\title{
Inclusão de fontes de óleo na dieta de cabras em lactação: produção, composição e perfil dos ácidos graxos do leite ${ }^{1}$
}

\author{
Fábio José Maia ${ }^{2}$, Antonio Ferriani Branco ${ }^{3}$, Gisele Fernanda Mouro ${ }^{4}$, Sabrina Marcantonio \\ Coneglian $^{5}$, Geraldo Tadeu dos Santos ${ }^{3}$, Tathiane Fernanda Minella ${ }^{6}$, Kátia Cylene Guimarães ${ }^{4}$ \\ 1 Parte da dissertação de Mestrado do primeiro autor. Financiamento CNPq. \\ ${ }^{2}$ Mestre em Zootecnia pela UEM. Bolsista CAPES. \\ ${ }^{3}$ Departamento de Zootecnia da UEM - Av. Colombo, 5790, CEP: 87020-900, Maringá - PR. Pesquisador Bolsista do CNPq. \\ ${ }^{4}$ Doutora em Zootecnia pela UEM. \\ ${ }^{5}$ Doutoranda em Zootecnia - UEM. \\ ${ }^{6}$ Graduação em Zootecnia - UEM. Bolsista de Iniciação Científica - CNPq.
}

RESUMO - O objetivo neste trabalho foi avaliar o efeito da inclusão de fontes de óleo na dieta de cabras em lactação sobre a produção, a composição e o perfil dos ácidos graxos da gordura do leite. Foram utilizadas quatro cabras da raça Saanen $(54 \pm 1,02 \mathrm{~kg} \mathrm{PV})$ aos 80 dias em lactação, canuladas no rúmen. Os tratamentos consistiram de dietas suplementadas com diferentes fontes de óleo (canola, arroz e soja) e de uma dieta controle. O delineamento experimental utilizado foi o quadrado latino $4 \mathrm{x} 4$ e os dados foram analisados utilizando-se o procedimento MIXED do SAS (2001). As diferenças entre as médias dos tratamentos foram determinadas por contrastes ortogonais, a 10\% de significância. A produção e composição do leite, à exceção do percentual de sólidos totais, não foram influenciadas pela suplementação lipídica na dieta. A utilização de óleos vegetais aumentou as concentrações de ácido linoléico conjugado (CLA), 18:0, 18:1n-9, 18:2, na gordura do leite. Constatou-se diminuição das concentrações de ácidos graxos saturados (AGS) e de cadeias curta (AGCC) e média (AGCM). Houve aumento das concentrações de ácidos graxos mono (AGMS) e poliinsaturados (AGPI), de ácidos graxos de cadeia longa, de ômega-3 e 6 e da relação AGPI/AGS A inclusão do óleo de soja na dieta, em comparação à suplementação com óleo de canola, resultou em maior concentração de AGPI e n-6, em maior relação de AGPI/AGS e n-6/n-3 e menor de 22:6n-4 no leite. O enriquecimento de dietas para cabras com óleos vegetais melhorou a qualidade do leite elevando a concentração de ácidos graxos desejáveis.

Palavras-chave: ácidos graxos insaturados, ácidos graxos saturados, óleos insaturados

\section{Feeding vegetable oil to lactating goats: milk production and composition and milk fatty acids profile}

\begin{abstract}
The objectives of this trial were to evaluate the effect of dietary oil supplementation on milk yield, milk composition and milk fatty acids profile of lactating Saanen goats. Four ruminally cannulated multiparous goats averaging $54 \pm 1.02 \mathrm{~kg} \mathrm{BW}$ and 80 days in milking were used. Goats were fed a control diet or a diet supplemented with one the following oil sources: canola, rice or soybean. The statistical design was a 4 x 4 Latin square and data were analyzed using the MIXED procedure of SAS (2001). Mean comparisons were done by orthogonal contrasts with significance level at $10 \%$. Except for content of milk total solids, no significant differences were observed for milk yield and milk composition by feeding different oil sources to lactating goats. Oil supplementation increased milk fat concentration of 18:0, 18:1n-9 and 18:2 (CLA) and decreased that of saturated fatty acids (SFA), short chain fatty acids (SCFA) and medium chain fatty acids (MCFA). Oil supplementation also increased milk concentration of monounsaturated (MUFA) and polyunsaturated fatty acids (PUFA), long chain fatty acids (LCFA), n-3, n-6 and the PUFA/SFA ratio. Feeding soybean oil to lactating goats increased milk content of PUFA, n-6, and the ratios of n-6/n-3 and PUFA/SFA and decreased concentration of 22:6n-4 compared to canola oil. Inclusion of vegetable oils in diet of lactating goats improved milk nutritional quality due to increased concentration of desirable fatty acids.
\end{abstract}

Key Words: saturated fatty acids, unsaturated fatty acids, unsaturated oil

\section{Introdução}

Suplementos lipídicos são incluídos na dieta de ruminantes para aumentar sua densidade energética, melhorar a utilização de nutrientes, incrementar as produções de carne e leite e possibilitar a manipulação da composição em ácidos graxos destes produtos (Palmquist et al., 1993). Um dos aspectos amplamente estudados é a composição dos ácidos graxos em produtos de origem animal, principalmente quanto ao seu enriquecimento por meio do aumento da concentração do ácido linoléico conjugado (CLA). 
Nos tecidos animais, são encontrados predominantemente dois isômeros dos ácidos graxos C18:2 (cis-9, trans-11 e trans-10, cis-12). Por isso, as espécies ruminantes e seus produtos são considerados as fontes alimentares mais ricas em CLA (Chin et al., 1992). Segundo Chin et al. (1994 a,b), o CLA é originalmente formado a partir da isomerização microbiana do ácido linoléico dietético, sendo um intermediário no curso de conversão do ácido linoléico a ácido oléico. A enzima responsável por esta reação é a ácido linoléico isomerase, que isomeriza o ácido linoléico preferencialmente para as formas cis-9 e trans-11 (Parodi, 1997). Assim, a classificação do ácido linoléico conjugado refere-se a um conjunto de oito possíveis isômeros posicionais e geométricos do ácido octadecadienóico (18:2), com duplas ligações conjugadas (Kelly et al., 1998).

O principal interesse no aumento da concentração de CLA no leite e nos demais produtos alimentares de origem animal é que este ácido graxo, além de apresentar comprovadamente propriedades antimutagênicas e anticarcinogênicas (Ha et al., 1987), atua na redução de agentes citotóxicos existentes nas células cancerígenas (Parodi, 1994). Além disso, o CLA apresenta ainda uma série de outras características benéficas à saúde, pois desencadeia estímulos de resposta imune contra a aterosclerose (Lee et al., 1994), apresenta propriedade hipocolesterolêmica (Kelly \& Bauman, 1996) e atividades na prevenção de outras doenças como diabetes (Houseknecht et al., 1998) e obesidade, além de atuar como um poderoso antioxidante (Park et al., 1999).

A concentração típica de CLA na gordura do leite é de 3 a $6 \mathrm{mg} / \mathrm{g}$, porém, podem ocorrer grandes variações entre os rebanhos leiteiros (Kelly \& Bauman, 1996). De modo geral, a concentração de CLA na gordura do leite é dependente da presença de ácidos graxos insaturados na dieta (Griinari et al., 1996). Animais ruminantes alimentados com grãos e/ou forragens secas ou ensiladas apresentam consideravelmente menos CLA em seus tecidos e produtos finais que animais alimentados com forragens verdes (Jiang et al., 1996).

Segundo Palmquist et al. (1993), o teor e a composição da gordura do leite são mais afetados pela quantidade e pelo tipo de gordura da dieta que por qualquer outro componente. Jiang et al. (1996) verificaram variação de 2,5 a 17,7 mg de $\mathrm{CLA} / \mathrm{g}$ de ácidos graxos no leite e sugeriram que esse ácido graxo pode ser diretamente aumentado por meio da dieta.

A produção e composição do leite, assim como suas características físico-químicas, são elementos passíveis de alterações, conforme a inclusão de gorduras na dieta, principalmente quando se trata de fontes ricas em ácidos graxos insaturados, por seu efeito inibitório sobre os microrganismos gram-positivos (Van Nevel \& Demeyer, 1988).
A composição lipídica do leite de cabra é a característica de maior importância na determinação de sua qualidade nutricional e comercial, pois esses componentes estão envolvidos tanto na produção como na qualidade de queijos e estão diretamente relacionados à coloração e ao sabor de produtos lácteos (Delacroix-Buchet \& Lamberet, 2000).

A vantagem nutricional do leite de cabra em relação ao de vaca consiste no menor tamanho dos glóbulos de gordura, que resulta em produtos mais facilmente digestíveis. Além disso, aproximadamente $20 \%$ dos ácidos graxos do leite de cabras são de cadeia curta $(\mathrm{C} 4: 0$ - C12:0) e são de rápida digestão (Jenness, 1980).

Assim, realizou-se este trabalho com o objetivo de avaliar os efeitos da inclusão de fontes de óleo, no nível de $5,1 \%$, na dieta de cabras Saanen em lactação sobre a produção e composição do leite e o perfil de ácidos graxos do leite.

\section{Material e Métodos}

O experimento foi conduzido no período de outubro de 2002 a janeiro de 2003 no Setor de Nutrição de Ruminantes da Fazenda Experimental de Iguatemi, no distrito de Iguatemi, e as análises foram realizadas no Laboratório de Análises de Alimentos e Nutrição Animal do Departamento de Zootecnia e no Laboratório de Análise de Alimentos do Departamento de Química, ambos pertencentes à Universidade Estadual de Maringá.

Foram utilizadas quatro cabras lactantes Saanen com 80 dias em lactação, canuladas no rúmen, pesando $54 \pm 1,02 \mathrm{~kg}$

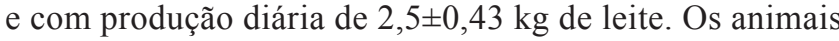
foram alojados individualmente em gaiolas de metal com piso de madeira ripada, provida de bebedouro e comedouro individuais.

A alimentação foi fornecida em duas porções iguais, às 8 e 16h, e a água disponibilizada ad libitum em bebedouros individuais. As gaiolas e as cânulas foram lavadas duas vezes ao dia para manutenção do controle higiênico/sanitário. As cabras foram ordenhadas duas vezes ao dia, às $8 \mathrm{~h} 30$ e $16 \mathrm{~h} 30$.

Os animais receberam dieta balanceada para atender às exigências de mantença e lactação, segundo o AFRC (1993), com relação volumoso:concentrado de 50:50. O volumoso foi composto de silagem de milho e o concentrado, de aveia, milho, farelo de soja, farelo de algodão, uréia, calcário e suplemento mineral. Os tratamentos avaliados consistiram da suplementação com três fontes de óleo (canola, arroz e soja) na dieta, em nível de 5,1\%, e de um tratamento controle sem a inclusão de óleo, como descrito a seguir:

$\mathrm{TT}=$ sem adição de fonte de óleo;

TOA $=$ adição de $5,1 \%$ de óleo de arroz na dieta total; 
TOC $=$ adição de $5,1 \%$ de óleo de canola na dieta total;

TOS = adição de $5,1 \%$ de óleo de soja na dieta total.

A composição dos alimentos empregados na formulação das dietas experimentais encontra-se na Tabela $1 \mathrm{e}$ a das rações experimentais na Tabela 2.

Foram estabelecidos períodos experimentais com 14 dias de duração, dos quais dez foram destinados à adaptação dos animais às dietas e o restante à realização das coletas. Segundo Scott et al. (1971), quatro dias são suficientes para induzir mudanças no perfil de ácidos graxos da gordura do leite de ruminantes a partir da suplementação com óleos na dieta.

A pesagem do leite foi realizada diariamente, porém, somente os dados do período entre o 8 o e o $14^{\circ}$ dias foram utilizados para avaliação das dietas.

Tabela 1 - Composição química dos alimentos utilizados nas dietas experimentais (\% na MS)

Table 1 - Chemical composition of feeds used in the formulation of the experimental diets (\% dry matter)

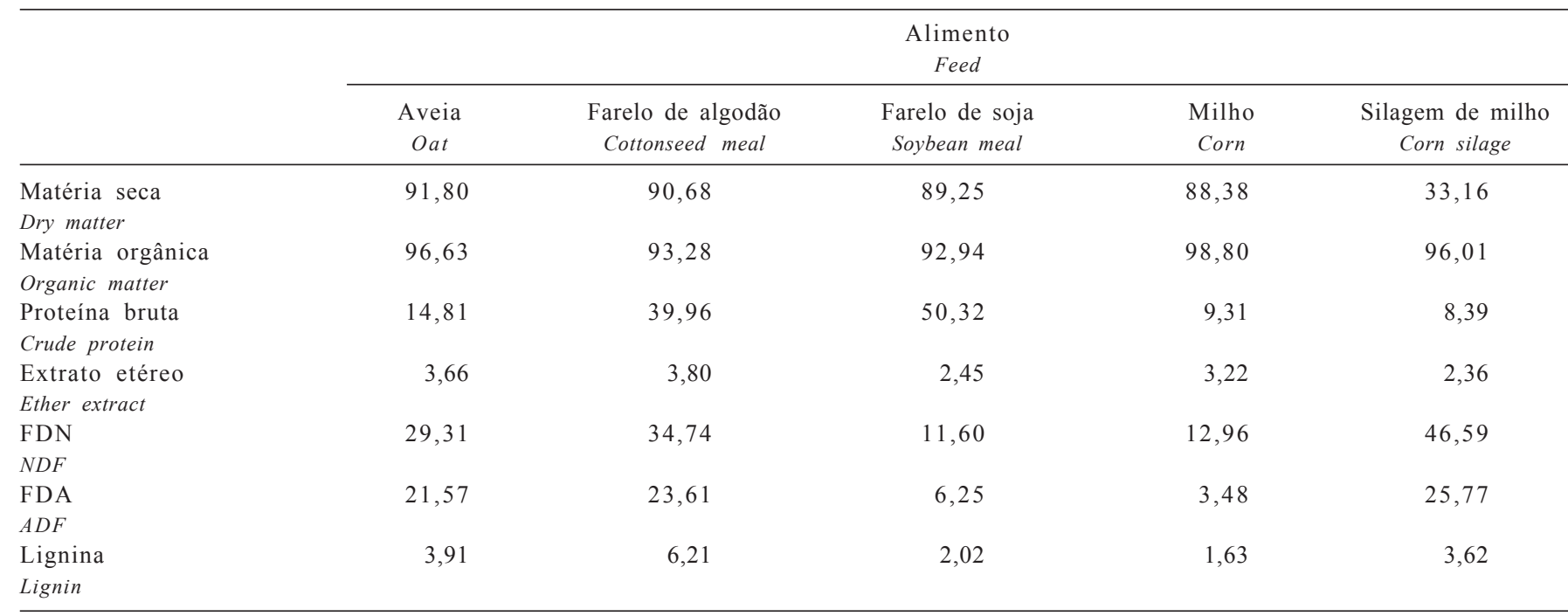

Tabela 2 - Composições percentual e química das dietas experimentais (\% na MS)

Table 2 - Ingredient and chemical compositions of the experimental diets (\% dry matter)

\begin{tabular}{|c|c|c|c|c|}
\hline \multirow[t]{2}{*}{$\begin{array}{l}\text { Alimento } \\
\text { Feed }\end{array}$} & \multicolumn{4}{|c|}{$\begin{array}{l}\text { Tratamento } \\
\text { Treatment }\end{array}$} \\
\hline & $\mathrm{TT}^{1}(\%)$ & $\mathrm{TOA}^{2}(\%)$ & $\operatorname{TOC}^{3}(\%)$ & $\operatorname{TOS}^{4}(\%)$ \\
\hline Aveia (Oat) & 13,55 & 10,90 & 10,90 & 10,90 \\
\hline Farelo de algodão (Cottonseed meal) & 10,45 & 10,45 & 10,45 & 10,45 \\
\hline Farelo de soja (Soybean meal) & 10,45 & 10,45 & 10,45 & 10,45 \\
\hline Uréia (Urea) & 0,30 & 0,50 & 0,50 & 0,50 \\
\hline Suplemento mineral (Mineral supplement) & 0,70 & 0,70 & 0,70 & 0,70 \\
\hline Calcário (Limestone) & 1,00 & 1,00 & 1,00 & 1,00 \\
\hline \multirow{2}{*}{\multicolumn{5}{|c|}{$\begin{array}{l}\text { Composição química } \\
\text { Chemical composition }\end{array}$}} \\
\hline & & & & \\
\hline Extrato etéreo (Ether extract) & 2,97 & 7,83 & 7,83 & 7,83 \\
\hline Cálcio (Calcium) & 0,66 & 0,65 & 0,65 & 0,65 \\
\hline Fósforo total (Total phosphorus) & 0,45 & 0,43 & 0,43 & 0,43 \\
\hline
\end{tabular}

1 TT = Sem adição de óleo na dieta (control diet).

2 TOA = Adição de $5,1 \%$ de óleo de arroz na dieta (rice oil).

3 TOC = Adição de $5,1 \%$ de óleo de canola na dieta (canola oil).

4 TOS = Adição de 5,1\% de óleo de soja na dieta (soybean oil).

${ }^{5}$ Nutrientes digestíveis totais (Total digestible nutrients): estimado NRC (1996).

${ }^{6}$ Proteína degradada no rúmen (Rumen-degradable protein): estimado NRC (1996). 
As amostras utilizadas na determinação da composição do leite foram coletadas durante três dias de cada período experimental, do $11^{\circ} \underline{0}$ ao $13^{\underline{o}}$ dia, duas vezes ao dia, em horários regulares, às 08 h30 e 16h30. Para preservação das características físico-químicas, foram conservadas com 2-bromo2-nitropropano-1,3 diol (BRONOPOL) e refrigeradas a $4^{\circ} \mathrm{C}$. As amostras coletadas pela manhã e à tarde foram eqüitativamente misturadas, constituindo amostras compostas para cada dia. Nas amostras compostas do leite, foram determinados os teores de EE e nitrogênio total, calculado pelo procedimento de Kjeldahl, descrito por Barbano et al. (1990). O congelamento das amostras de leite, como citado anteriormente, inviabilizou a execução de qualquer técnica disponível para a quantificação do percentual de gordura.

A extração e análise do perfil de ácidos graxos da gordura do leite foram feitas conforme relatado por Murphy \& McNeill (1995), por congelamento, descongelamento e centrifugação.

Amostras de aproximadamente $250 \mathrm{~mL}$ de leite foram mantidas por uma noite em congelador $\left(-18^{\circ} \mathrm{C}\right) \mathrm{e}$, em seguida, foram descongeladas até atingir temperatura ambiente, sendo transferidas para tubos apropriados e centrifugadas a $3.000 \mathrm{rpm}$ por 30 minutos para separação da fração gordurosa.

Os ésteres metílicos de ácidos graxos foram obtidos por meio da transesterificação dos triacilgliceróis, conforme método 5509 da ISO (1978), em solução de n-heptano e $\mathrm{KOH} /$ metanol. Foram tranferidos aproximadamente $200 \mathrm{mg}$ da matéria graxa para tubo de ensaio com tampa rosqueável e capacidade para $10 \mathrm{~mL}$, onde foram adicionados $2,0 \mathrm{~mL}$ de n-heptano. O material foi agitado até solubilização total da matéria graxa, sendo adicionado de 2,0 mL de solução $2 \mathrm{~mol} / \mathrm{L}$ de $\mathrm{KOH}$ em metanol e agitado vigorosamente por mais 5 minutos. Após separação das fases, o sobrenadante contendo os ésteres metílicos de ácidos graxos foi cuidadosamente pipetado, transferido para tubo eppendorff e armazenado em congelador $\left(-18^{\circ} \mathrm{C}\right)$ até a realização das análises.

Consta na Tabela 3 a composição para os principais ácidos graxos dos óleos vegetais adicionados às dietas experimentais.

As análises dos ésteres metílicos dos ácidos graxos da gordura do leite foram realizadas em cromatógrafo a gás 14-A (Shimadzu, Japão), equipado com detector de ionização de chama (FID) e coluna capilar de sílica fundida com $100 \mathrm{~m}$ de comprimento, 0,25 $\mathrm{mm}$ de diâmetro interno e 0,20 $\mu \mathrm{m}$ de cianoalquil polisiloquixano, CP-Sil 88 (Chrompack, EUA). Para registro das concentrações dos ácidos graxos, o aparelho foi acoplado a um Integrador Processador CG-300
(Instrumentos Científicos CG). As condições adotadas no processo de separação cromatográfica foram:

Temperatura do injetor: $220^{\circ} \mathrm{C}$;

Temperatura da coluna: $60^{\circ} \mathrm{C}$ mantida por 4 minutos, $60^{\circ} \mathrm{C}$ para $140^{\circ} \mathrm{C}, 40^{\circ} \mathrm{C} / \mathrm{min} ; 140^{\circ} \mathrm{C}$ mantida por 10 minutos; $140^{\circ} \mathrm{Ca} 225^{\circ} \mathrm{C}, 5^{\circ} \mathrm{C} / \mathrm{min}$, mantida por 12 minutos.

Temperatura do detector: $240^{\circ} \mathrm{C}$;

Vazão dos gases: - gás de arraste: 1,2 mL/min $\left(\mathrm{H}_{2}\right)$;

$$
\begin{aligned}
& \text { - Make-up: } 30 \mathrm{~mL} / \mathrm{min}\left(\mathrm{N}_{2}\right) \text {; } \\
& \text { - hidrogênio: } 30 \mathrm{~mL} / \mathrm{min} ; \\
& \text { - ar sintético: } 300 \mathrm{~mL} / \mathrm{min} \text {. }
\end{aligned}
$$

Volume injetado: - 0,5 $\mu \mathrm{L}$ de amostra em duplicata

Os picos dos ácidos graxos foram identificados por comparação ao seu tempo de retenção, utilizando-se uma mistura de padrões Sigma (EUA). A quantificação dos ácidos graxos foi feita utilizando-se fatores de correção para as áreas de pico, calculados a partir de misturas padrões de ácidos graxos. Determinadas as concentrações, os ácidos graxos foram agrupados em ácidos graxos de cadeia curta (AGCC - até 10 carbonos), ácidos graxos de cadeia média (AGCM - de 11 a 16 carbonos) e ácidos graxos de cadeia longa (AGCL - acima de 16 carbonos). Os dados foram analisados em um quadrado latino 4 x 4 utilizando-se o procedimento MIXED do SAS (2001). As fontes de

Tabela 3 - Composição percentual dos principais ácidos graxos dos óleos vegetais utilizados na formulação das dietas experimentais

\begin{tabular}{|c|c|c|c|}
\hline \multirow[t]{2}{*}{$\begin{array}{l}\text { Ácido graxo } \\
\text { Fatty acid }\end{array}$} & \multicolumn{3}{|c|}{$\begin{array}{c}\text { Óleo } \\
\text { Oil }\end{array}$} \\
\hline & $\begin{array}{c}\text { Canola } \\
\text { Canola }\end{array}$ & $\begin{array}{c}\text { Arroz } \\
\text { Rice }\end{array}$ & $\begin{array}{c}\text { Soja } \\
\text { Soybean }\end{array}$ \\
\hline $16: 0$ & 5,29 & 18,43 & 11,53 \\
\hline $18: 0$ & 2,64 & 1,76 & 3,94 \\
\hline $18: 1$ & 61,99 & 43,00 & 24,83 \\
\hline $18: 2 n-6$ & 24,48 & 35,28 & 55,62 \\
\hline $18: 3 n-3$ & 5,60 & 1,61 & 4,08 \\
\hline $\operatorname{AGS}(S F A)$ & 7,93 & 20,19 & 15,47 \\
\hline AGI (UFA) & 92,07 & 79,89 & 84,53 \\
\hline AGMI (MUFA) & 61,99 & 43,00 & 24,83 \\
\hline AGPI (PUFA) & 30,08 & 36,89 & 59,70 \\
\hline$n-3$ & 5,60 & 1,61 & 4,08 \\
\hline$n-6$ & 24,48 & 35,28 & 55,62 \\
\hline AGPI/AGS $(P U F A) /(S F A)$ & 3,79 & 1,83 & 3,86 \\
\hline$n-6 / n-3$ & 4,37 & 21,91 & 13,63 \\
\hline
\end{tabular}

Table 3 - $\quad$ Percentual composition of main fatty acids of vegetable oils used in the experimental diets formulation

Resultados expressos em porcentagem do total de ácidos graxos representam médias de análises, em triplicatas, de todos os valores obtidos. AGS = total de ácidos graxos saturados; $A G I=$ total de ácidos graxos insaturados; $\mathrm{AGMI}=$ total de ácidos graxos monoinsaturados; $\mathrm{AGPI}=$ total de ácidos graxos poliinsaturados; $n-6$ = total de AG ômega-6; n-3 = total de AG ômega-3.

Values obtained from triplicate analyses and expressed as percentage of total milk fatty acids. SFA = saturated fatty acids; UFA = unsaturated fatty acids; MUFA = monounsaturated fatty acids; $P$ UFA = polyunsaturated fatty acids. 
variação consideradas no modelo foram animal, período e tratamento. As diferenças entre as médias dos tratamentos foram determinadas por contrastes ortogonais, a $10 \%$ de significância. Os contrastes ortogonais avaliados foram controle $\mathrm{x}$ tratamentos (cont $\mathrm{x}$ trat), óleo de arroz x óleo de sementes de oleaginosas (arr x ole) e óleo de canola x óleo de soja (can x soj).

O modelo matemático adotado na análise dos dados foi o seguinte:

$$
\mathrm{Y}_{\mathrm{ijk}}=\mu+\mathrm{T}_{\mathrm{i}}+\mathrm{A}_{\mathrm{j}}+\mathrm{P}_{\mathrm{k}}+\mathrm{e}_{\mathrm{ijk}}
$$

em que $\mathrm{Y}_{\mathrm{ijk}}=$ resposta observada, $\mu=$ constante geral, $\mathrm{T}_{\mathrm{i}}=$ efeito do tratamento $i$, variando de 1 a $4 ; \mathrm{A}_{\mathrm{j}}=$ efeito do animal $j$, variando de 1 a $4 ; \mathrm{P}_{\mathrm{k}}=$ efeito do período $k$, variando de 1 a 4 ; e $\mathrm{e}_{\mathrm{ijk}}=$ erro aleatório associado a cada observação $i j k$.

\section{Resultados e Discussão}

As estimativas prévias de consumo (2,02 kg MS/dia) e EM (4,83 Mcal/dia), realizadas conforme o AFRC (1993) para uma cabra com produção média diária de $2 \mathrm{~L}$, foram confirmadas pelos dados obtidos; em que a produção de leite foi de 2,47 $\pm 0,09 \mathrm{~kg} / \mathrm{dia}$, a partir do consumo de 2,02 $\pm 0,06$ $\mathrm{kg} \mathrm{MS} / \mathrm{dia}$, o que representou consumo de energia metabolizável (EM) de 4,83 $\pm 0,15 \mathrm{Mcal} /$ dia.

Os dados de produção ( $\mathrm{kg} /$ dia) e composição percentual em relação aos sólidos totais e PB do leite são descritos na Tabela 4.

A produção de leite e os teores de $\mathrm{PB}(\mathrm{g} /$ dia) e proteína (\%) do leite não foram influenciados $(\mathrm{P}>0,10)$ pela suplementação lipídica na dieta. Do mesmo modo, não houve diferenças entre óleos de oleaginosas (canola e soja) e de arroz nem entre as duas oleaginosas. Os resultados para estas variáveis confirmam os obtidos por Pereira et al.
(1993) e Rabello (1995), em vacas, e Lu (1993) e Sleiman et al. (1998), em cabras, os quais não notaram alterações no teor de proteína do leite. Porém, Oliveira Jr. et al. (2002) verificaram efeito quadrático na produção ( $\mathrm{g} / \mathrm{dia})$ e no teor de proteína (\%) em cabras alimentadas com adição de grãos de soja níveis crescentes na dieta para atingir valores de EE de 3,2 a $6,3 \%$ da MS da dieta.

A inclusão de $7 \%$ de lipídios na dieta não afetou a produção de leite e a concentração de lactose, proteína, gordura e sólidos totais do leite (Santos et al., 2001). Esses dados confirmam os achados de Vargas et al. (2002), que não verificaram influência da inclusão de lipídios na dieta (níveis de 3 e 7\% da MS) sobre a produção de leite e sua composição em proteína.

A concentração de sólidos totais (\%) no leite foi superior $(\mathrm{P}<0,01)$ quando os animais foram alimentados com as dietas suplementadas $(11,9 ; 11,6$ e $11,7 \%$ para os óleos de arroz, canola e soja, respectivamente) em relação ao tratamento controle (10,8\%). No entanto, a produção de sólidos totais ( $\mathrm{g} / \mathrm{dia})$ não foi alterada $(\mathrm{P}>0,10)$ pela inclusão de óleos à dieta. Não houve diferença entre óleos de oleaginosas (canola e soja) e de arroz nem entre ambas as oleaginosas para concentração (\%) e produção de sólidos totais (g/dia).

O aumento verificado no teor de sólidos totais (\%), equivalente a $8,3 \%$, pode ser atribuído a uma possível elevação do teor de gordura do leite, uma vez que não foram encontradas alterações no conteúdo de proteína e que a concentração de lactose dificilmente é influenciada por modificações das fontes utilizadas na formulação de dietas para animais em lactação (Mir et al., 1999; Vargas et al., 2002; Giesy et al., 2002).

A resposta à suplementação lipídica difere consideravelmente entre cabras (Chilliard \& Bocquier, 1993), vacas

Tabela 4 - Médias e coeficientes de variação (CV\%) para a produção de leite (PL) e sua composição em sólidos totais (ST) e PB Table 4 - Means and coefficients of variation for milk production (MP) and content and yield of milk total solids (TS) and milk crude protein (CP)

\begin{tabular}{|c|c|c|c|c|c|c|c|c|}
\hline & \multicolumn{4}{|c|}{$\begin{array}{c}\text { Tratamento } \\
\text { Treatment }\end{array}$} & \multirow[t]{2}{*}{ CV (\%) } & \multicolumn{3}{|c|}{$\begin{array}{c}\text { Contraste } \\
\text { Contrast }\end{array}$} \\
\hline & $\mathrm{T} \mathrm{T}^{1}$ & $\mathrm{TOA}^{2}$ & $\mathrm{TOC}^{3}$ & $\mathrm{TOS}^{4}$ & & Cont $\times$ Trat $^{5}$ & Arr $\times \mathrm{Ole}^{6}$ & Can $x$ Soj $^{7}$ \\
\hline $\mathrm{ST}(T S)(\%)$ & 10,84 & 11,86 & 11,62 & 11,73 & 2,27 & $* * *$ & 0,29 & 0,56 \\
\hline $\mathrm{ST}(T S)(\mathrm{g} / \mathrm{dia})$ & 283,49 & 294,72 & 274,99 & 288,04 & 8,04 & 0,86 & 0,38 & 0,45 \\
\hline $\mathrm{PB}(C P)(\%)$ & 2,97 & 3,01 & 2,95 & 3,06 & 5,35 & 0,74 & 0,94 & 0,39 \\
\hline
\end{tabular}

1 TT = sem adição de óleo na dieta (control diet).

2 TOA = adição de $5,1 \%$ de óleo de arroz na dieta total (rice oil).

3 TOC $=$ adição de $5,1 \%$ de óleo de canola na dieta total (canola oil).

4 TOS = adição de $5,1 \%$ de óleo de soja na dieta total (soybean oil).

5 Controle $x$ tratamentos (control $x$ oil sources).

6 Óleo de arroz x óleo de sementes de oleaginosas (rice oil x oily seeds).

7 Óleo de canola $x$ óleo de soja (canola oil $x$ soybean oil).

*** Valores de $\mathrm{P}<0,01$ 
leiteiras (Chilliard et al., 2001) e ovelhas (Nudda et al., 2002): a produção de leite no meio da lactação aumenta em vacas, mas não em cabras e ovelhas; a concentração de gordura do leite aumenta em cabras e ovelhas, porém pode não alterar em vacas; o teor de $\mathrm{PB}$ do leite diminui em vacas e ovelhas, mas se altera em cabras; a secreção de PB não muda em vacas e cabras, mas diminui em ovelhas. Portanto, os dados encontrados neste trabalho corroboram os descritos por esses autores.

Na Tabela 5 são apresentados os dados referentes à composição percentual dos principais ácidos graxos da gordura do leite.
A concentração do ácido butírico (4:0) não foi alterada com a inclusão de óleos à dieta e não diferiu entre óleos de oleaginosas (canola e soja) e de arroz nem entre essas oleaginosas. Segundo Young \& Gibson (1994), o ácido butírico pode ser importante na prevenção de tumores, inibindo a uroquinase, um facilitador da entrada de células malignas no substrato das células.

Contudo, as concentrações dos ácidos capróico (6:0), caprílico (8:0), cáprico (10:0), láurico (12:0), mirístico (14:0), pentadecílico (15:0), palmítico (16:0) e margárico (17:0) na gordura do leite reduziram $(\mathrm{P}<0,09)$ com a inclusão de óleos

Tabela 5 - Composição percentual dos ácidos graxos na gordura do leite de cabras Saanen alimentadas com dietas acrescidas de diferentes fontes de óleo

Table 5 - Milk fatty acids profile of lactating Saanen dairy goats supplemented with different dietary oil sources

\begin{tabular}{|c|c|c|c|c|c|c|c|c|}
\hline \multirow[t]{2}{*}{$\begin{array}{l}\text { Ácido graxo } \\
\text { Fatty acid }\end{array}$} & \multicolumn{4}{|c|}{$\begin{array}{c}\text { Tratamento } \\
\text { Treatment }\end{array}$} & \multirow[t]{2}{*}{ CV (\%) } & \multicolumn{3}{|c|}{$\begin{array}{c}\text { Contraste } \\
\text { Contrast }\end{array}$} \\
\hline & $\mathrm{T} \mathrm{T}^{1}$ & $\mathrm{TOA}^{2}$ & $\mathrm{TOC}^{3}$ & $\mathrm{TOS}^{4}$ & & Cont $\times$ Trat $^{5}$ & Arr $x \mathrm{Ole}^{6}$ & Can $x \operatorname{Soj}^{7}$ \\
\hline $4: 0$ & 0,68 & 0,77 & 0,71 & 0,67 & 9,62 & 0,32 & 0,88 & 0,41 \\
\hline $6: 0$ & 1,18 & 1,09 & 0,98 & 1,02 & 11,94 & 0,09 & 0,34 & 0,69 \\
\hline $8: 0$ & 1,82 & 1,57 & 1,29 & 1,42 & 22,68 & 0,09 & 0,39 & 0,64 \\
\hline $10: 0$ & 8,46 & 5,16 & 4,69 & 4,58 & 18,28 & $* * *$ & 0,45 & 0,89 \\
\hline $12: 0$ & 4,08 & 2,23 & 2,11 & 2,27 & 29,50 & $* * *$ & 0,95 & 0,77 \\
\hline $14: 0$ & 10,40 & 6,39 & 5,92 & 5,84 & 16,58 & $* * *$ & 0,53 & 0,93 \\
\hline $15: 0$ & 0,79 & 0,54 & 0,52 & 0,53 & 13,81 & $* * *$ & 0,83 & 0,90 \\
\hline $16: 0$ & 29,19 & 24,50 & 19,59 & 21,76 & 13,10 & $* * *$ & 0,09 & 0,36 \\
\hline $16: 1 \mathrm{n}-7$ & 0,49 & 0,37 & 0,32 & 0,39 & 28,79 & 0,09 & 0,82 & 0,47 \\
\hline $17: 0$ & 0,61 & 0,32 & 0,39 & 0,38 & 18,51 & $* * *$ & 0,23 & 0,93 \\
\hline $17: 1 \mathrm{n}-9$ & 0,36 & 0,15 & 0,19 & 0,15 & 49,76 & 0,02 & 0,73 & 0,64 \\
\hline $18: 0$ & 10,34 & 16,75 & 19,74 & 17,53 & 16,85 & $* * *$ & 0,30 & 0,29 \\
\hline $18: 1 \mathrm{n}-9$ & 25,67 & 33,41 & 37,01 & 35,31 & 7,24 & $* * *$ & 0,11 & 0,35 \\
\hline $18: 1 \mathrm{n}-5$ & 0,42 & 0,46 & 0,57 & 0,79 & 35,33 & 0,15 & 0,12 & 0,17 \\
\hline $18: 2 n-6$ & 3,62 & 3,44 & 3,42 & 4,50 & 12,47 & 0,56 & 0,12 & 0,02 \\
\hline $18: 3 n-6$ & 0,34 & 0,50 & 0,56 & 0,52 & 20,23 & 0,26 & 0,20 & 0,54 \\
\hline $18: 3 n-3$ & 0,23 & 0,27 & 0,24 & 0,24 & 24,88 & 0,56 & 0,42 & 0,96 \\
\hline 18:2 (CLA) & 0,91 & 1,43 & 1,10 & 1,70 & 30,19 & 0,08 & 0,63 & 0,14 \\
\hline $20: 5 n-3$ & 0,25 & 0,25 & 0,23 & 0,18 & 38,71 & 0,54 & 0,39 & 0,44 \\
\hline $22: 6 n-3$ & 0,16 & 0,41 & 0,41 & 0,23 & 41,06 & 0,04 & 0,28 & 0,09 \\
\hline $\operatorname{AGS}(S F A)$ & 67,55 & 59,31 & 55,95 & 56,00 & 4,68 & $* * *$ & 0,11 & 0,98 \\
\hline AGI (UFA) & 32,45 & 40,69 & 44,05 & 44,00 & 6,94 & $* * *$ & 0,09 & 0,98 \\
\hline AGMI (MUFA) & 26,94 & 34,39 & 38,09 & 36,64 & 7,65 & $* * *$ & 0,11 & 0,46 \\
\hline AGPI (PUFA) & 5,51 & 6,30 & 5,96 & 7,36 & 9,33 & 0,02 & 0,36 & 0,01 \\
\hline$n-6$ & 3,97 & 3,94 & 3,98 & 5,02 & 10,33 & 0,21 & 0,08 & 0,02 \\
\hline$n-3$ & 0,64 & 0,93 & 0,88 & 0,65 & 24,31 & 0,51 & 0,57 & 0,30 \\
\hline AGPI/AGS & 0,08 & 0,11 & 0,11 & 0,13 & 12,76 & $* * *$ & 0,21 & 0,05 \\
\hline$n-6 / n-3$ & 6,31 & 4,53 & 5,11 & 8,25 & 34,71 & 0,79 & 0,15 & 0,08 \\
\hline $\mathrm{AGCC}(S C F A)$ & 12,14 & 8,58 & 7,64 & 7,69 & 16,00 & $* * *$ & 0,35 & 0,99 \\
\hline AGCM (MCFA) & 44,95 & 34,02 & 28,46 & 30,79 & 9,52 & $* * *$ & 0,07 & 0,38 \\
\hline $\mathrm{AGCL}(L C F A)$ & 42,90 & 57,40 & 63,86 & 61,53 & 6,60 & $* * *$ & 0,06 & 0,55 \\
\hline
\end{tabular}

1 TT = sem adição de óleo na dieta (control diet).

2 TOA = adição de $5,1 \%$ de óleo de arroz na dieta total (rice oil).

3 TOC $=$ adição de $5,1 \%$ de óleo de canola na dieta total (canola oil).

4 TOS $=$ adição de $5,1 \%$ de óleo de soja na dieta total (soybean oil).

5 Controle $\mathrm{x}$ tratamentos (control $\mathrm{x}$ oil sources).

6 Óleo de arroz x óleo de sementes de oleaginosas (rice oil x oily seeds).

7 Óleo de canola $x$ óleo de soja (canola oil $x$ soybean oil).

8 AGS = ácidos graxos saturados (saturated fatty acids).

$9 \mathrm{AGI}=$ ácidos graxos insaturados (unsaturated fatty acids).

$10 \mathrm{AGMI}=$ ácidos graxos monoinsaturados (monounsaturated fatty acids).

$11 \mathrm{AGPI}=$ ácidos graxos poliinsaturados (polyunsaturated fatty acids).

12 AGCC = ácidos graxos de cadeia curta (short chain fatty acids).

$13 \mathrm{AGCM}=$ ácidos graxos de cadeia média (medium chain fatty acids).

${ }^{14} \mathrm{AGCL}=$ ácidos graxos de cadeia longa (long chain fatty acids).

*** Valores de $\mathrm{P}<0,01$ 
vegetais à dieta. De acordo com Romo et al. (2000), em vacas, a infusão abomasal de uma mistura de isômeros cis e trans do C18:1 resultou na síntese de gordura do leite com menores proporções do C14:0, C14:1, C16:0 e C16:1.

A concentração de ácido palmítico (Tabela 5) no leite das cabras foi maior $(\mathrm{P}<0,09)$ com a suplementação com óleo de arroz $(24,5 \%)$ que com óleos de oleaginosas $(20,68 \%)$.

Menores proporções de ácidos graxos de cadeias curta e média foram encontradas no leite de para vacas submetidas a infusão no abomaso (Gaynor et al., 1994) ou sob suplementação com o ácido graxo transoctadecenóico (Griinari et al., 1998), o que indica redução da síntese de novo de ácidos graxos pela glândula mamária (Grummer, 1991). Este efeito foi evidenciado neste trabalho, em que o enriquecimento da dieta com fontes de óleos ricos em AGPI promoveu a redução da maior parte dos ácidos de cadeias curta e média.

A inclusão de óleos vegetais na dieta promoveu aumento $(\mathrm{P}<0,01)$ da concentração do ácido esteárico (18:0) na gordura do leite, que passou de $10,34 \%$ no tratamento controle para 19,$74 ; 16,75$ e 17,53\% nos tratamentos com óleo de canola, arroz e soja, respectivamente. $\mathrm{O}$ aumento na concentração do ácido esteárico correspondeu em média a $74 \%$ quando comparados o tratamento controle e as dietas suplementadas. Contudo, não houve diferenças entre óleos de oleaginosas (canola e soja) e de arroz nem entre as duas oleaginosas $(\mathrm{P}>0,10)$.

De Peters et al. (2001) encontraram maiores concentrações para o C18:0 quando vacas receberam óleo de canola na dieta ou infusão direta no rúmen. Esses autores relataram que este fato pode ocorrer como conseqüência da biohidrogenação ruminal dos ácidos graxos insaturados presentes no óleo de canola. Os óleos de arroz, canola e soja, cujas concentrações de ácidos graxos insaturados são 79,89; 92,07 e 84,53\% (Tabela 3), respectivamente, podem ter ocasionado comportamento semelhante ao verificado por esses autores.

O aumento na concentração do ácido esteárico pode ter sido provocado pela extensa biohidrogenação ruminal dos ácidos graxos 18:1, 18:2n-6 e 18:3n-3, uma vez que, juntos, representavam aproximadamente 92,84 e 80\% dos lipídios dos óleos de canola, soja e arroz e que as concentrações de ácido esteárico na gordura do leite das cabras sob suplementação com estas fontes de lipídeos foram 19,74; 17,53 e 16,75\%, respectivamente. Parte do ácido esteárico é transformada em ácido oléico pela ação da enzima $\Delta-9$ desaturase na glândula mamária, que promove a insaturação do ácido esteárico (18:0) no carbono 9, originando o ácido oléico(18:1n-9).
A inclusão de óleos vegetais na dieta elevou $(\mathrm{P}<0,01)$ a concentração do ácido oléico (18:1n-9), de modo que o valor encontrado $(35,2 \%)$ foi $37 \%$ superior ao obtido com a dieta controle $(25,67 \%)$. Estes resultados corroboram os encontrados por De Peters et al. (2001), que verificaram aumento da concentração do ácido oléico (18:1n-9) nos tratamentos com óleo de canola quando comparados ao controle. Todavia, neste estudo, este efeito foi verificado não apenas com o uso do óleo de canola, mas também com as outras fontes utilizadas na elaboração das dietas.

As concentrações dos ácidos linoléico (18:2n-6) e linolênico (18:3n-3) na gordura do leite não foram influenciadas pela adição de óleos vegetais à dieta (cont x trat) Contudo, a gordura do leite dos animais alimentados com dietas contendo óleo de soja apresentou maior concentração $(\mathrm{P}<0,02)$ do ácido linoléico que a das cabras alimentadas com dietas enriquecidas com óleo de canola. De Peters et al. (2001) relataram que a composição da gordura do leite para o 18:2n-6 e 18:3n-3 foi maior em vacas que receberam infusão com óleo de canola no abomaso. A infusão abomasal preveniu a biohidrogenação ruminal dos AGI, disponibilizando maior quantidade pós-ruminal para a síntese de gordura do leite. A adição do óleo à dieta e a infusão ruminal, no entanto, não promoveram nenhum tipo de proteção contra a biohidrogenação dos AGPI.

A inclusão de fontes de óleos vegetais à dieta aumentou a concentração de CLA na gordura do leite $(\mathrm{P}<0,08)$. As dietas suplementadas propiciaram conteúdo de CLA $55 \%$ superior em relação ao tratamento controle, com médias de 0,$91 ; 1,10 ; 1,43$ e $1,70 \%$ para os tratamentos controle, óleo de canola, arroz e soja, respectivamente. O tratamento com óleo de soja merece maior destaque, pois, neste caso, a diferença na concentração de CLA em relação ao tratamento controle chega a aproximadamente $87 \%$ (Figura 1). Não foi observada diferença entre o óleo de arroz e o de oleaginosas.

A utilização de óleos em vez de sementes de oleaginosas, como fonte de lipídios adicionais à dieta, aumenta o nível de CLA, uma vez que o emprego deste tipo de suplemento propicia o ataque dos microrganismos sobre estas fontes de energia de forma mais efetiva, favorecendo o processo de biohidrogenação, ao contrário do que ocorre com as sementes de oleaginosas, em que a fonte de lipídio está protegida por uma matriz protéica que impede a biohidrogenação, reduzindo a formação dos principais precursores do CLA.

O CLA presente na gordura do leite é proveniente, em parte, da biohidrogenação ruminal do ácido linoléico e parte é resultante da atividade da enzima $\Delta-9$ desaturase nas células da glândula mamária, que transformam o ácido 
vacênico absorvido da corrente sangüínea em CLA (Bauman \& Griinari, 2001). Dessa forma, espera-se que, quanto maior a concentração do 18:2n-6 na dieta, maiores as chances de elevar a concentração de CLA na gordura do leite. Este comportamento foi evidenciado neste estudo, pois o óleo de soja foi a fonte mais rica em ácido linoléico, seguido dos óleos de arroz e de canola. Este mesmo comportamento foi verificado para a concentração percentual de CLA na gordura do leite (Figura 1).

Os acréscimos na concentração de CLA na gordura do leite foram de aproximadamente $21 \%$ (óleo de canola), $57 \%$ (óleo de arroz) e 87\% (óleo de soja). Mir et al. (1999), estudando os efeitos da adição de níveis crescentes de óleo de canola na dieta de cabras em lactação, verificaram que o percentual de CLA passou de 88 para $210 \%$ quando a inclusão de óleo passou de 2 para 4\%, respectivamente, em relação ao tratamento sem óleo. Contudo, não houve efeito benéfico no aumento do conteúdo de CLA com a inclusão de $6 \%$ de óleo de canola.

A maior parte do CLA encontrado no leite e nos tecidos é originária da atividade microbiana no rúmen. Entretanto, existem algumas evidências de que o CLA pode ser formado por meio de mecanismos endógenos a partir da desaturação do trans 18:1 (Mir et al., 1999).

Segundo Gulati et al. (1997), a quantidade de ácidos graxos transferidos da dieta para a gordura do leite está diretamente relacionada ao nível de proteção implementado à fonte suplementar de óleo e ao tipo de suplemento empregado. Portanto, a manipulação da dieta de cabras leiteiras, dependendo do objetivo proposto para o produto final, pode ser uma forma de controlar as características nutricionais e/ou físico-químicas da gordura do leite.

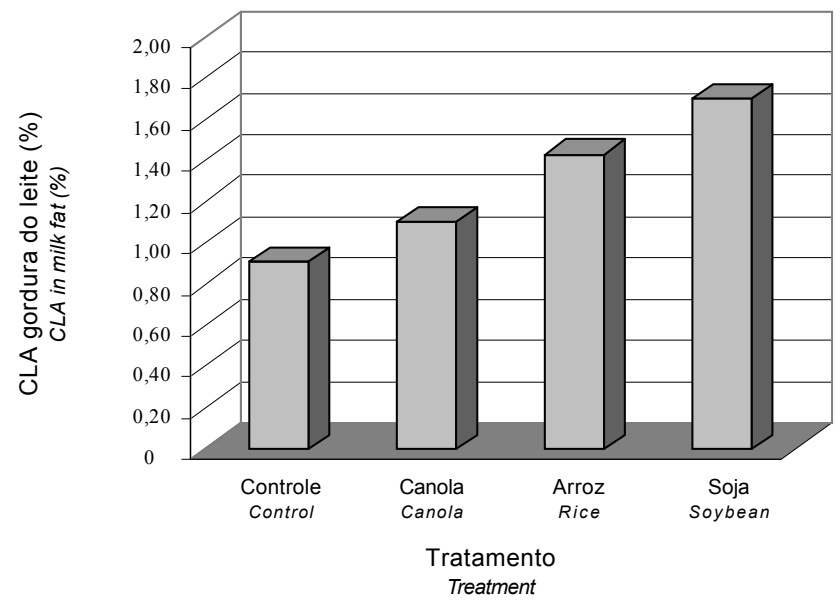

Figura 1 - Efeito da suplementação com fontes de óleos vegetais sobre a concentração de CLA na gordura do leite de cabras Saanen.

Figure 1 - Effect of dietary oil supplementation on content of milk CLA of Saanen goats.
A modificação das características da gordura dos produtos lácteos pode ser um recurso para melhorar sua imagem junto aos consumidores, que buscam produtos nutricionalmente mais saudáveis, que disponham de substâncias com propriedades comprovadamente terapêuticas, como o CLA. No entanto, por extrapolação direta em estudos desenvolvidos com ratos, Ip et al. (1991) estimaram que a ingestão diária de CLA por uma pessoa adulta com $70 \mathrm{~kg}$ deveria ser equivalente a $3,5 \mathrm{~g}$. Considerando um consumo diário de aproximadamente $200 \mathrm{~mL}$ de leite e que o leite de cabra apresenta em média 4\% de gordura, o consumo médio diário de CLA seria de aproximadamente $140 \mathrm{mg}$ (14\% do recomendado) para um indivíduo consumindo o leite de animais sob suplementação com óleo de soja.

A concentração de AGS na gordura do leite das cabras no tratamento controle foi superior $(\mathrm{P}<0,01)$ à obtida com suplementação, como resultado do alto nível de insaturação das fontes de óleos vegetais utilizadas na formulação das dietas. Porém, não houve diferença entre a suplementação com óleo de arroz e óleo de oleaginosas nem entre as oleaginosas (média de 57\% para estes tratamentos). Desse modo, pode-se afirmar que a inclusão de óleos vegetais ricos em AGPI na dieta de cabras leiteiras reduziu em $15,6 \%$ a concentração de ácidos graxos saturados quando adicionados em nível de 5,1\% da dieta. Concomitantemente à redução verificada na concentração de AGS, houve elevação $(\mathrm{P}<0,01)$ de $32 \%$ na concentração de AGI na gordura do leite quando as dietas foram enriquecidas com fontes de óleos vegetais. Tendência semelhante foi relatada por Santos et al. (2001), em pesquisa com vacas alimentadas com dietas contendo grão e óleo de soja. Neste estudo, o leite de cabras diferiu $(\mathrm{P}<0,09)$ entre os tratamentos com óleo de arroz (40,69\%) e óleos de oleaginosas (44,03\%).

A suplementação das dietas com fontes de óleos resultou em concentrações de AGMI superiores $(\mathrm{P}<0,01)$ às encontradas no tratamento controle, com valores de 26,94 (controle), 38,09 (óleo de canola) 34,39 (óleo de arroz) e 36,64\% (óleo de soja). A concentração dos AGPI seguiu a mesma tendência verificada para os AGMI, de modo que as dietas suplementadas resultaram em concentrações superiores $(\mathrm{P}<0,02)$ desta classe de lipídios em relação ao tratamento controle. Entre os óleos de oleaginosas, o de soja foi superior na elevação do nível de AGPI, aumentando sua concentração em $34 \%$, enquanto o óleo de canola elevou apenas $8 \%$ e o de arroz, $14 \%$.

A análise de contrastes revelou não ter havido diferença na concentração de ômega- 6 com a inclusão de fontes adicionais de óleo à dieta. Entretanto, óleos provenientes de sementes de oleaginosas aumentaram $(\mathrm{P}<0,08)$ a concentração deste componente em comparação ao óleo 
de arroz. Do mesmo modo, o aumento promovido pelo óleo de soja foi superior $(\mathrm{P}<0,02)$ ao do óleo de canola.

A concentração de ômega-3, assim como a relação n-6/n-3, não diferiu entre o tratamento controle e a suplementação com fontes de óleo. Também não houve diferença entre o óleo de arroz e as fontes de oleaginosas. No entanto, a dieta enriquecida com óleo de soja apresentou relação $n-6 / n-3$ superior $(\mathrm{P}<0,05)$ em comparação àquela enriquecida com óleo de canola. Existem ainda divergências entre os pesquisadores quanto à ingestão de ácidos graxos ômega-6 e ômega-3. Simopoulos et al. (1999) consideraram ideais para esta relação valores entre 5 e 10 . Portanto, todos os valores encontrados neste estudo estariam dentro da faixa. A relação AGPI/AGS foi maior $(\mathrm{P}<0,05)$ com a suplementação que no tratamento controle. Quanto ao comprimento da cadeia carbônica, a concentração de AGCC e de AGCM foi maior $(\mathrm{P}<0,05)$ para os animais do tratamento controle que para aqueles sob suplementação lipídica. Comportamento contrário foi observado para as concentrações de AGCL, pois a suplementação de lipídios à dieta elevou $(\mathrm{P}<0,05)$ as concentrações destes ácidos graxos na gordura do leite.

Pode-se afirmar que a suplementação da dieta com óleos vegetais seria uma forma eficiente de melhorar as propriedades nutricionais da gordura do leite, pois promoveu aumento na concentração de AGPI e na relação AGPI/AGS $(\mathrm{P}<0,01)$.

\section{Conclusões}

O enriquecimento da dieta de cabras leiteiras com óleos vegetais melhorou a qualidade nutricional do leite, promovendo o aumento na concentração dos ácidos graxos desejáveis na alimentação humana (os poliinsaturados) e melhorando a relação AGPI/AGS.

\section{Literatura Citada}

AGRICULTURAL AND FOOD RESEARCH COUNCIL - AFRC. Energy and protein requirements of ruminants. Wallington: CAB International, 1993. 159p.

BARBANO, D.M.; CLARK, J.L.; DUNHAM, C.E. et al. Kjeldahl method for determination of total nitrogen content of milk: Collaborative study. Journal of Association of Animal Chemistry, v.73, p.849-859, 1990.

BAUMAN, D.E.; GRIINARI, J.M. Regulation and nutritional manipulation of milk fat: low-fat milk syndrome. Livestock Production Science, v.70, p.15-29, 2001.

CHILLIARD, Y.; BOCQUIER, F. Effects of fat supplementation on milk yield and composition in dairy goats and ewes. In: INTERNATIONAL SYMPOSIUM LA QUALITA NELL PRODUZIONI DEI PICCOLI RUMINANTI, 5., 1993, Varese. Proceedings... Varese: Camera di Commercio Industria Artigianato Agricultura di Varese, 1993. p.61-78.
CHILLIARD, Y.; FERLAY, A.; DREAU, M. Effect of different types of forages, animal fat or marine oils in cow's diet on milk fat secretion and composition, specially conjugated linoleic acid (CLA) and polyunsaturated fat acids. Livestock Production Science, v.70, p.31-48, 2001.

CHIN, S.F.; LIU, W.; STORKSON, J.M. et al. Dietary sources of conjugated dienoic isomers of linoleic acid, a newly recognized class of anticarcinogens. Journal Food Composition and Analysis, v. 5, p.185-197, 1992.

CHIN, S.F.; STORKSON, J.M.; LIU, W. et al. Conjugated linoleic acid $(9,11$ and 10,12 octadecadienoic acid) is produced in conventional but not germ-free rats fed linoleic acid. Journal of Nutrition, v.124, p.694-701, 1994a.

CHIN, S.F.; STORKSON, J.M.; ALBRIGHT, K.J. et al. Conjugated linoleic acid is a growth factor for rats as show by enhanced weight gain and improved feed efficiency. Journal of Nutrition, v.124, p.2344-2349, 1994b.

DELACROIX-BUCHET, A.; LAMBERET, G. Sensorial properties and typicity of goat dairy products. In: INTERNATIONAL CONFERENCE ON GOATS, 7., 2000, Tours. Proceedings... Tours: 2000. p.559-563.

DePETERS, E.J.; CANT, J.P. Nutritional factors influencing the nitrogen composition of bovine milk: a review. Journal of Dairy Science, v.75, p.2043-2070, 1992.

DePETERS, E.J.; GERMAN, J.B.; TAYLOR, S.J. Fatty acid and triglyceride composition of milk fat from lactating Holstein cows in response to supplemental canola oil. Journal of Dairy Science, v. 84, p.929-936, 2001.

GAYNOR, P.J.; ERDMAN, R.A.; TETER, B.B. et al. Milk fat yield and composition during abomasal infusion of cis or trans octanodecenoates in Holstein cows. Journal of Dairy Science, n.77, p.157-165, 1994.

GIESY, J.G.; McGUIRE, M.A.; SHAFII, B. et al. Effect of dose calcium salts of conjugated linoleic acid (CLA) on percentage and fatty acid content of milk fat in midlactation Holstein cows. Journal of Dairy Science, v.85, p.20232029, 2002 .

GRIINARI, J.M; DWYER, D.A.; McGUIRE, M.A. et al. Partially hydrogenated fatty acids and milk fat depression. Journal of Dairy Science, v.79, p.177, 1996.

GRIINARI, J.M.; DWYER, D.A.; McGUIRE, D.A. et al. Transoctadecenoic acids and milk fat depression in lactating dairy cows. Journal of Dairy Science, v.81, p.1251-1261, 1998.

GRUMMER, R.R. Effect of feed on the composition of milk fat Journal of Dairy Science, v.74, p.3244-3257, 1991.

GULATI, S.K.; BYERS, E.B.; BYERS, Y.G. Effect of feeding different fat supplements on the fatty acid composition of goat milk. Animal Feed Science Technology, v.66, p.159-164, 1997.

HA, Y.L.; GRIMM, N.K.; PARIZA, M.W. Anticarcinogens from fried ground beef: heat altered derivatives of linoleic acid. Carcinogenesis, v. 8, p.1881-1887, 1987.

HOUSEKNECHT, K.L.; VANDENHEUVEL, J.P.; MOYACAMERINA, S.Y. et al. Dietary conjugated linoleic acid normalizes impaired glucose tolerance in the Zucker diabetic fatty falfa rat. Biochemical and Biophysical Research Communications, v.244, p. 678-682, 1998.

INTERNATIONAL ORGANIZATION FOR STANDARDIZATION - ISO. Animal and vegetable fats and oils - Preparation of methyl esters of fatty acids. Geneve: ISO. Method ISO 5509. p.1-6. 1978.

IP, C.; CHIN, S.F.; SCIMECA, J.A. et al. Mammary cancer prevention by conjugated dienoic derivative of linoleic acid. Cancer Research, v.51, p.6118-6124, 1991.

JENNESS, R. Composition and characteristics of goats milk: review 1968-1979. Journal of Dairy Science, v.79, p.438-445, 1980.

JIANG, J.; BJOERCK, L.; FONDEN, R. et al. Occurrence of conjugated cis-9, trans-11 octadecenoic acid in bovine milk: effects of feed and dietary regimen. Journal of Dairy Science, v.79, p.438-445, 1996. 
KELLY, M.L.; BAUMAN, D.E. Conjugated linoleic acid: a potent anticarcinogen found in milk fat. In: CORNELL NUTRITIONAL CONFERENCE, 1996, Ithaca. Anais... Ithaca: 1996. p.68-74.

KELLY, M.L.; BERRY, J.R.; DWYER, D.A. et al. Dietary fatty acid sources affect conjugated linoleic acid concentrations in milk from lactating dairy cows. Journal of Nutrition, v.128, p.881-885, 1998.

LEE, K.N.; KRITCHEVSKY, D.; PARIZA, M.W. Conjugated linoleic acid and atherosclerosis in rabbits. Atherosclerosis, v.108, p. 19-25, 1994.

LU, C.D. Implication of feeding isoenergetic diets containing animal fat on milk composition of Alpine does during early lactation. Journal of Dairy Science, v.76, p.1137-1147, 1993.

MIR, Z.; GOONEWARDENE, L.A.; OKINE E. et al. Effect of feeding canola oil on constituents, conjugated linoleic acid (CLA) and long chain fatty acids in goats milk. Small Ruminant Research, v.33, p.137-143, 1999.

MURPHY, J.J.; McNEILL, G.P. Effects on milk fat composition and cow performance of feeding concentrates containing full fat rapessed and maize distillers grains on grass-silage based diets. Livestock Production Science, v.44, p.1-11, 1995.

NUDDA, A.; BATTACONE, G.; BENCINI, R. et al. Nutrition and milk quality. In: PULINA, G. (Ed.) Dairy sheep feeding and nutrition. Bologna: Avenue Media, 2002. p.197-228.

OLIVEIRA JR., R.C.; SISIN, I.; PIRES, A.V. et al. Desempenho de cabras em lactação com grão de soja. Acta Scientiarum, v.24, p.1113-1118, 2002.

PALMQUIST, D.L.; BEAULIEU, D.; BARBANO, D.M. Feed and animal factors affecting milk fat composition. Journal of Dairy Science, v.76, p.1753-1771, 1993.

PARODI, P.W. Conjugated linoleic acid: an anticarcinogenic fatty acid present in milk. Australian Journal of Dairy Technology, v.49, p.93-97, 1994.

PARK, Y.; STORKSON, J.M.; ALBRIGHT, K.J. et al. Evidence that the trans-10, cis-12 isomer of conjugated linoleic acids induces body composition changes in mice. Lipids, v.34, p.235$241,1999$.

PEREIRA, C.M.A. Grãos de soja moído na ração de vacas em lactação. 2. Produção e composição do leite. Revista Brasileira de Zootecnia, v.27, p.1225-1233, 1993.

RABELLO, T.G.; VALADARES FILHO, S.C.; COELHO DA SILVA, J.F. et al. Grão de soja moído na alimentação de vacas em lactação. I. Consumos, produção e composição do leite. Revista Brasileira de Zootecnia, v.25, p.345-356, 1996.
ROMO, G.A.; ERDMAN, R.A.; TETER, B.B. et al. Milk composition and apparent digestibilities of dietary fatty acids in lactating dairy cows abomasally infused with cis or trans fatty acids. Journal of Dairy Science, v.83, p.2609-2619, 2000 .

SANTOS, F.L.; SILVA, M.T.C.; LANA, R.P. et al. Efeito da suplementação de lipídios na ração sobre a produção de ácido linoléico conjugado (CLA) e a composição da gordura do leite de vacas. Revista Brasileira de Zootecnia, v.30, p.19311938, 2001.

STATISTICAL ANALYSIS SYSTEM - SAS. SAS user's guide: estatistics. Eletronic version 8.1. Cary: 2001 (CD-ROM).

SCOTT, T.W.; COOK, L.J.; MILLS, S.C. Protection of dietary polyunsaturated fatty acids against microbial hydrogenation in ruminants. Journal American Oil Chemical Society, v.8, p.358-364, 1971 .

SIMOPOULOS, A.P.; LEAF, A.; SALEM, N. Essentiality and recommended dietary intakes for omega- 6 e omega- 3 fatty acids. Annals of Nutrition and Metabolism, v.43, p.127$130,1999$.

SLEIMAN, E.T.; BAYDOUN, M.I.; UWAYJAN, M.G. et al Influence of feeding calcium protected fat on goat milk production and composition. Journal of Animal Science, v.76. (Suppl. 1), p.302, 1998.

Van NEVEL, C.J.; DEMEYER, D.I. Manipulation of ruminal fermentation. In: HOBSON, P.N. (Ed.) The rumen microbial ecosystem. Essex: Elsevier Science Publishers, 1988. p.387-443.

VARGAS, L.H.; LANA, R.P.; JHAM, G.N. et al. Adição de lipídios na ração de vacas leiteiras: parâmetros fermentativos ruminais, produção e composição do leite. Revista Brasileira de Zootecnia, v.31, p.522-529, 2002.

WONSIL, B.J.; HERBEIN, J.H.; WATKINS, B.A. Dietary and ruminally derived trans-18:1 fat acids alter bovine milk lipids. Journal Nutrition, v.124, p.556-565, 1994.

YOUNG, G.P.; GIBSON, P.R. Butyrate and the colorectal cancer cell. In: SHORT CHAIN FATTY ACIDS, 1994, London. Proceedings... London: 1994. p.148-160. 